\title{
Noninvasive Treatment Approach of Radial Pseudoaneurysm
}

\author{
Syed Iftikhara, c, Asma Jamila, Javad Savoj ${ }^{\mathrm{a}}$, Patrick $\mathrm{Hu}^{\mathrm{b}}$
}

\begin{abstract}
Transradial approach for cardiac catheterization is a viable alternative to transfemoral approach given its ease of access, lessened complication risk, and post procedural comfort for patients. Radial pseudoaneurysm presents as a rare complication in less than $1 \%$ of these procedures. The use of external compression banding is an approach that shows promise as a noninvasive attempt towards resolving this complication. However, it has been documented in very few reports. We describe a case of an 82-year-old woman who underwent transradial approach to cardiac catheterization, and developed a radial pseudoaneurysm following the procedure as confirmed by Doppler ultrasonography. We used compressive banding as a technique to attempt to resolve this radial pseudoaneurysm. Following a strict protocol of pneumatic banding, repeat ultrasonography revealed complete resolution of radial pseudoaneurysm. This case highlights a potentially noninvasive technique that could serve as a first-line approach towards resolving this rare phenomenon.
\end{abstract}

Keywords: Transradial approach; Radial pseudoaneurysm; Noninvasive treatment

\section{Introduction}

The use of radial access for cardiac catheterization has increasingly become the approach of choice for many interventional cardiologists. As the transradial approach continues to increase in utilization, potential complications will be discovered and discussed, and standardized treatment modalities have to be developed to address them. One such rare complication that has been observed is that of a radial pseudoaneurysm. In contrast to the well-known femoral pseudoaneurysm, radial

Manuscript submitted February 23, 2019, accepted March 16, 2019

aDepartment of Internal Medicine, UC Riverside School of Medicine/Riverside Community Hospital, Riverside, CA 92501, USA

bDepartment of Cardiology, UC Riverside School of Medicine/Riverside Community Hospital, Riverside, CA 92501, USA

${ }^{\mathrm{c} C}$ Corresponding Author: Syed Iftikhar, Department of Internal Medicine, UC Riverside School of Medicine/Riverside Community Hospital, Riverside, CA 92501, USA. Email: Syed.iftikhar.md@gmail.com

doi: https://doi.org/10.14740/cr850 pseudoaneurysms are a highly uncommon presentation following cardiac catheterization. The RIVAL trial reported an incidence of radial pseudoaneurysm requiring intervention at less than $0.002 \%$ of all cardiac catheterizations [1]. This rare complication has no standardized treatment modality yet to be developed to address it. We describe a case of a patient who developed radial pseudoaneurysm, and document one method of successful treatment by noninvasive means, via external compression device.

\section{Case Report}

An 82-year-old woman with hypertension and atrial fibrillation on warfarin presented with $2 \mathrm{~h}$ of acute onset epigastric pain with radiation to the chest, jaw, and left arm. Initial vitals were within normal limits. She was in moderate distress. Labs were significant for a subtherapeutic international normalized ratio (INR). Electrocardiogram revealed ST segment elevations in the inferolateral leads. After Allen's test was done, she was deemed an appropriate candidate for approach via right radial artery and taken for urgent cardiac catheterization. The patient was found to have an acute thrombus in the distal posterior descending artery (PDA) and posterior left ventricular (PLV) branch. She was treated with angioplasty and aspiration thrombectomy, and the acute thrombi were thought to be secondary to thromboembolic phenomenon. The patient received dual antiplatelet therapy following the procedure, and a pneumatic band was placed over the patient's right wrist and remained in place until it was removed per protocol. The following day, patient reported pain at the right wrist. On exam, a pulsatile $2.4-\mathrm{cm}$ mass with an overlying bruit was noted. A color flow Doppler ultrasound study was performed and it revealed a radial artery pseudoaneurysm (Fig. 1). Initial discussions with vascular surgery, and interventional radiology did not yield a consensus approach towards resolving this complication. Nonspecific compression via pneumatic band was attempted for $18 \mathrm{~h}$; however this was initially proved unsuccessful as seen on repeat Doppler. Following this, a different compressive approach was attempted. Pneumatic band was inflated until radial pulse flow was occluded as evidenced by absence of pulse oximetry wave form. The level of occlusion was achieved with $12 \mathrm{cc}$ of air inflation. Air was removed in small segments until radial artery flow was noted (this was noted to be at $10 \mathrm{cc}$ of air inflation of the pneumatic band). Upon dialogue with nursing staff, it was discussed that the 


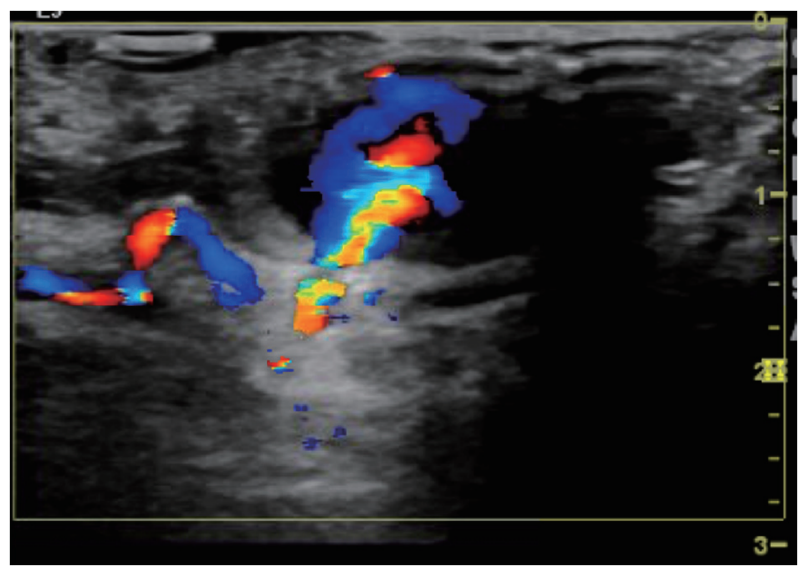

Figure 1. Duplex ultrasound demonstrating patent right radial artery with a pseudoaneurysm just measuring $2.3 \mathrm{~cm} \times 2.4 \mathrm{~cm} \times 1.4 \mathrm{~cm}$, and the neck of the pseudoaneurysm measuring $2.1 \mathrm{~mm}$.

pneumatic band should remain in place at current level of compression for $1 \mathrm{~h}$ and then $1 \mathrm{cc}$ of air should be removed each hour until device was fully deflated (Table 1). After the allotted time course of $10 \mathrm{~h}$ that it took to deflate the pneumatic band, a repeat arterial Doppler ultrasound revealed complete resolution of the radial pseudoaneurysm (Fig. 2). She also reported improvement of pain, and was discharged that day.

\section{Discussion}

While pseudoaneurysm is a well-known complication in transfemoral coronary intervention procedures with incidence ranging from $0.2-3 \%$, it is extremely rare when a radial approach is used. Incidence of radial artery pseudoaneurysm after catheterization has been reported as low as $0.009 \%$ [2].

Known risk factors for radial pseudoaneurysm include the use of glycoprotein IIb/IIIa inhibitors and an elevated body mass index. Very elderly patients may also be at increased risk. Other potential factors that can be hypothesized from the more

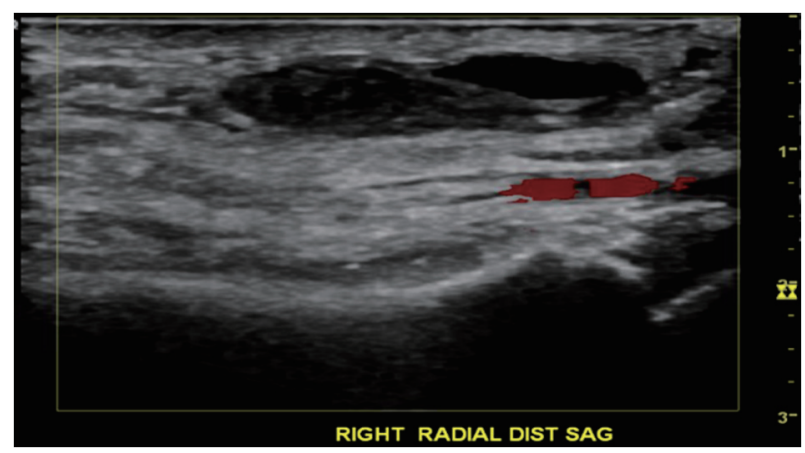

Figure 2. Duplex ultrasound now showing resolution of the pseudoaneurysm with a patent radial artery and superficial hematoma measuring $2.7 \mathrm{~cm} \times 0.9 \mathrm{~cm} \times 2.7 \mathrm{~cm}$.

common femoral artery pseudoaneurysm include use of larger sheaths, periprocedural use of antiplatelet agents, use of anticoagulants, and hypertension [3].

Risks of radial artery pseudoaneurysms have not been systematically reviewed, and however, in theory they present risks similar to those of femoral artery pseudoaneurysms. Some of these potential risks include rupture or distal limb ischemia [4].

Treatment of radial pseudoaneurysm has not been standardized. Treatment options range from percutaneous thrombin injection, surgical repair, sonographically guided compression, external compression devices and close monitoring for spontaneous resolution. Many of these techniques have been recorded in various journals, and many of them have shown success; however some of them carry more significant risks than others. For example, surgical approach has been documented but due to the small area of defect, various approaches may be entertained. For small neck aneurysm ligation alone may be sufficient. However, excision of the vessel wall with vein patch angioplasty may be recommended for large neck aneurysms [3]. Also due to its limited occurrence, it is quite possible that many surgeons will not be comfortable performing such procedures. For these reasons, it is reasonable that surgical approach should be considered a last line of treatment.

Another approach that has been described is thrombin in-

Table 1. Size of Pseudoaneurysm at Different Time Points

\begin{tabular}{lll}
\hline Time $(\mathbf{h})$ & Inflated air $(\mathbf{m L})$ in compression device & Size of pseudoaneurysm \\
\hline 0 & 10 & $2.5 \mathrm{~cm}$ \\
1 & 9 & Not measured \\
2 & 8 & Not measured \\
3 & 7 & Not measured \\
4 & 6 & Not measured \\
5 & 5 & Not measured \\
6 & 4 & Not measured \\
7 & 3 & Not measured \\
8 & 2 & Not measured \\
9 & 1 & Not measured \\
10 & 0 & 0 cm \\
\hline
\end{tabular}


jection into the pseudoaneurysm with intent to produce immediate thrombosis [5]. This, however, has not been successfully reproduced, and requires a highly specialized interventional team, which in many locales is unfeasible. Furthermore this approach carries the significant risk of arterial embolization and peripheral thrombosis of the affected limb.

Because of the difficulty in addressing radial pseudoaneurysm via surgical approach, as well as the risks involved with injectable approach, there has been a need to evaluate noninvasive means towards addressing this phenomenon. A replicable and noninvasive approach has not been effectively designed, although a few reports have highlighted potential attempts. One such maneuver is that of direct compression at the neck of the pseudoaneurysm using a transducer probe. The proposed mechanism behind this maneuver is that probe compression stimulates stasis of blood and promotes coagulation and occlusion internally within the pseudoaneurysm [6]. This has been shown to be an effective method; however one institution reported success rate of only 74\% [7]. Furthermore, the downside of this method included extreme discomfort from prolonged compression, high cost in terms of workload for medical staff, use of sophisticated technical equipment and total procedure time [8].

The appeal for an effective, noninvasive approach towards radial pseudoaneurysms is clear. Because of the time and staff commitments with the aforementioned transducer probe approach, a more practical approach is ideal. A unique strategy of utilizing a Terumo band (TR Band ${ }^{\circledR}$ ) to apply consistent radial compression has been utilized successfully in a few case studies $[9,10]$. This approach is highly replicable, requires less staffing and time than other procedures, is based upon literature review, and has been successful. Due to these reasons, if repeatedly replicated, such a method holds promise as a potential first-line option for those who develop this complication, especially those with small to moderate sized radial arteries [11]. We attempted external compression with the TR band based upon two reports of its efficacy in the literature $[8,9]$. The TR band is a transparent plate that is positioned over the pseudoaneurysm and is secured via a Velcro strap. The inflator syringe permits accurate pressure adjustment as air is injected into the side port which inflates the compression balloon overlaying the pseudoaneurysm. It is important to perform modified Allen's test or Barbeau's test prior to this maneuver, as dual arterial flow to hand is essential because the radial artery will have a brief period of compression and thus limitation of blood flow. After placing a pulse oximeter on patient's first finger, we inflated a TR band with $12 \mathrm{cc}$ of air and monitored for absence of wave form on pulse oximetry. This was deemed maximal pressure point and air was removed until waveform returned at $10 \mathrm{cc}$ of air. Each hour, we asked the nurse to deflate $1 \mathrm{cc}$ of air from the TR band until completely deflated. We maintained pressure for approximately 10 $\mathrm{h}$ with this method and repeated Doppler ultrasound described resolution. This is a technique which is similar to other strategies described; however deflation intervals were set at longer periods. Our interval for deflating $1 \mathrm{cc}$ of air was $1 \mathrm{~h}$, while other studies deflated air every $15 \mathrm{~min}$ [9]. This approach was enacted for practical approaches (nursing staff ability to assist with deflation at a more feasible time interval) as well as for ensuring adequate compression to allow resolution. There was no neurologic or vascular sequelae observed. Similarly, a case has been described in which a similar concept of compressive banding has been utilized using a HemoBand (HemoBand Co., Portland, OR, USA) [4]. Thus, this shows a degree of replicability despite what sort of compressive banding device is available at each specific institution.

\section{Conclusions}

Arterial access sites should be evaluated frequently following arterial catheterization, especially in patients with risk factors for radial pseudoaneurysm. Early identification and treatment of pseudoaneurysm is imperative in order to reduce the risk of rupture. This case highlights a potentially important solution to a rare but novel problem which may become more common given the higher number of cardiac catheterization procedures which are approached via radial route. External compression via TR band and similar compressive devices provides a reasonable, affordable and noninvasive approach that can be used before embarking on more invasive treatment methods such as surgery and percutaneous thrombin injection.

\section{Acknowledgments}

None to declare.

\section{Financial Disclosure}

None to declare.

\section{Conflict of Interest}

None to declare.

\section{Informed Consent}

Informed consent has been obtained.

\section{Author Contributions}

SI, AJ, JS and PH all have contributed to this work.

\section{References}

1. Jolly SS, Yusuf S, Cairns J, Niemela K, Xavier D, Widimsky P, Budaj A, et al. Radial versus femoral access for coronary angiography and intervention in patients with acute coronary syndromes (RIVAL): a randomised, parallel group, multicentre trial. Lancet. 2011;377(9775):14091420 . 
2. Tosti R, Ozkan S, Schainfeld RM, Eberlin KR. Radial artery pseudoaneurysm. J Hand Surg Am. 2017;42(4):295 e291-295 e296.

3. Hamid T, Harper L, McDonald J. Radial artery pseudoaneurysm following coronary angiography in two octogenarians. Exp Clin Cardiol. 2012;17(4):260-262.

4. Nazer B, Boyle A. Treatment of recurrent radial artery pseudoaneurysms by prolonged mechanical compression. J Invasive Cardiol. 2013;25(7):358-359.

5. Komorowska-Timek E, Teruya TH, Abou-Zamzam AM, Jr., Papa D, Ballard JL. Treatment of radial and ulnar artery pseudoaneurysms using percutaneous thrombin injection. J Hand Surg Am. 2004;29(5):936-942.

6. Bhat T, Teli S, Bhat H, Akhtar M, Meghani M, Lafferty J, Gala B. Access-site complications and their management during transradial cardiac catheterization. Expert Rev Cardiovasc Ther. 2012;10(5):627-634.

7. Eisenberg L, Paulson EK, Kliewer MA, Hudson MP,
DeLong DM, Carroll BA. Sonographically guided compression repair of pseudoaneurysms: further experience from a single institution. AJR Am J Roentgenol. 1999;173(6):1567-1573.

8. Liou M, Tung F, Kanei Y, Kwan T. Treatment of radial artery pseudoaneurysm using a novel compression device. J Invasive Cardiol. 2010;22(6):293-295.

9. Cauchi MP, Robb PM, Zemple RP, Ball TC. Radial artery pseudoaneurysm: a simplified treatment method. J Ultrasound Med. 2014;33(8):1505-1509.

10. Kongunattan V, Ganesh N. Radial artery pseudoaneurysm following cardiac catheterization: a nonsurgical conservative management approach. Heart Views. 2018;19(2):6770 .

11. Ghanavati R, Arab Ahmadi M, Behnam B. Successful nonsurgical treatment of a radial artery pseudoaneurysm following transradial coronary angiography. J Tehran Heart Cent. 2017;12(2):82-84. 